\title{
Fearfully and Wonderfully Made: An Evo-Devo Perspective on Human Anatomy
}

\author{
Quirks of Human Anatomy: An Evo-Devo Look at the Human Body, by Louis I. Held, Jr. \\ Cambridge University Press: New York, Pp. Xii + 260, P/b \$34.99
}

\author{
Andrew J. Petto
}

Published online: 10 October 2009

(C) Springer Science + Business Media, LLC 2009

\begin{abstract}
A typical lesson for beginning students in the visual arts is to take one half of a photograph of a human face and then to draw the other half. The irony is that the students who are the best at copying the half image usually fail to create a portrait that "looks like" the person in the photograph. This is because humans, as other bilateran species, are mostly symmetrical. Quirks of Human Anatomy illustrates the genetic basis and developmental pathways underlying what gross anatomists have come to label "fluctuating asymmetries"and traces the evolutionary history of those processes. Author Louis Held brings readers a rich and extensively documented exploration of the evolutionary and developmental bases of modern human anatomy. As is frequently the case in developmental studies, Quirks takes some of its clues on developmental processes and pathways from studies of anatomical errors and defects, but this book offers so much more than just a morbid anatomy of human development. Instead, Quirks examines defects and errors as a way of understanding what happens when things go right. Perhaps the most significant characteristic of this book is that it extends the "quirks" of human anatomy to find their evolutionary roots.
\end{abstract}

Quirks of Human Anatomy has much to recommend it. From my perspective as a teacher of anatomy, I rejoice any time that an exploration of the human form makes clear the evolutionary basis of our biological features. However, Quirks hits the jackpot by delivering on the other two components that contribute to our current form: development and function. A second strength of this book is that it provides detailed-

\section{A. J. Petto $(\square)$}

Department of Biological Sciences,

University of Wisconsin-Milwaukee,

P.O. Box 413, Milwaukee, WI 53201-0413, USA

e-mail: ajpetto@uwm.edu and extensively illustrated and documented-accounts of developmental genetics and pathways focused around the sorts of questions that beginning students in anatomy might have: "How did this thing get to be this way in human anatomy?" Sometimes, the answer is in the events that caused the separation of humans from the other apes; but sometimes, the answer is in events that happened hundreds of millions of years earlier.

The illustrations are very helpful. They show how developmental processes produce the results they do-and why certain types of malformations are more common than others. However, what might be even more valuable are the author's reflections. These show the sense of awe that scientists feel when confronting the natural processes capable of producing "endless forms most beautiful" from what are essentially a few simple rules. And they show that this sense of wonder about the natural world is not diminished by understanding how things work.

I heartily recommend this book and found it a joy to read. However, there are a few minor quibbles. Every so often, the author drops out of his very clear and carefully crafted narrative to interject a conversational phrase, "Can you imagine that?" Although relatively rare, these Dave-Barryesque interjections are distracting, and they detract from the power of the writing.

In most cases, I would argue that the author is answering "how" questions and not "why" questions - at least not the sorts of "why" questions that relate to purpose or meaning. We really do not know why, for example, nodal is expressed the way it is in chordates compared to other deuterosomes to produce the particular asymmetrical arrangement of internal organs (p. 27-30); even though this expression is why we observe the specific expressions of the anatomical features that we do in these organisms. We do know how nodal produces these features - at least in 
a general way, if not the details in every case (e.g., lung asymmetry, p. 30). So, because the term "why" can have both the meanings of purpose and of process, it would be better to make it clear that these answers are about process, not purpose.

Finally, I would argue that the "quirks" of the title are not just about human anatomy. Held acknowledges this early in the book: "Humans, it turns out, are no more quirky than other species" (p. 54). The book is full of examples of evo-devo studies of a wide variety of species. The takehome message for the quirks of their anatomy is the same as for humans. I suppose, however, that Quirks of Drosophilan Anatomy would probably not appeal to as many readers as Quirks of Human Anatomy, and this is a book that deserves the widest possible readership.

The power of this book is that it shows how the human form is the result of the interplay of developmental, environmental, and evolutionary processes - not as a "just so" story that fabricates conditions that human anatomical features are "adapted for" but as an exploration of the biological processes that make us who we are anatomically. It also demonstrates aptly that for every developmental process that we have been able to unravel, there is not one that is designed especially for producing the unique features of human anatomy. Instead, as evolutionary science would predict, the processes and their components are modified from those that existed in ancestral populations and pressed into service in new contexts with new outcomes.

There is surely much, much more to learn; Held is candid about that. But so far, the expectations from evolutionary theory that even the unique features of human anatomy would have their roots deep in our phylogenetic heritage are entirely fulfilled. "The larger lesson is that Homo sapiens, like most species, is just one still frame in an epic movie of anatomical ebb and flow that is playing itself out at a glacially slow place on a geological timescale" (p. 128). The take-home lesson is the same as for nearly every biological inquiry conducted in an evolutionary context: We are part of life and biologically continuous with the rest of nature. The rich array of evo-devo research that is brought to bear in Quirks of Human Anatomy provides another independent line of evidence for our connection to the tree of life. 